\title{
Performance Analysis of the Quality of Service- aware Networking Scheme for Smart Internet of Things Gateways
}

\author{
Anderson Augusto Simiscuka \\ School of Electronic Engineering \\ Dublin City University \\ anderson.simiscuka2@mail.dcu.ie
}

\author{
Marija Bezbradica \\ School of Computing \\ Dublin City University \\ marija.bezbradica@dcu.ie
}

\author{
Gabriel-Miro Muntean \\ School of Electronic Engineering \\ Dublin City University \\ gabriel.muntean@dcu.ie
}

\begin{abstract}
The extremely large number of devices available to the modern day user, with the increase in device intercommunication, is fuelling the latest Internet of Things (IoT) development. IoT needs to enable exchange of various types of data, from sensor data to multimedia, between numerous diverse devices differing in power, connectivity, mobility and energy, while also maintaining high levels of Quality of Service (QoS). This paper performs statistical analysis of the innovative NETworking Scheme for sMart IoT gatewayS (NETSMITS) in terms of several QoS network-related metrics with most significant impact on devices' performance. NETSMITS introduces an innovative algorithm which uses QoS and service relevance metrics in order to efficiently cluster intercommunicating IoT objects. Statistical analysis is performed on the QoS data collected in a highly relevant multi-device scenario in order to understand NETSMITS' behaviour. Interesting results were obtained, describing the relationship between the QoS metrics and different types of IoT devices.
\end{abstract}

Keywords-Internet of Things (IoT), statistics, QoS.

\section{INTRODUCTION}

The Internet of Things (IoT) enables large number of devices, sensors and appliances to access the Internet for multiple purposes, including offering and consuming different services. Service-wise IoT is expected to have a total economic impact of $\$ 3.9$ trillion to $\$ 11.1$ trillion a year by 2025 [1]. This is additionally driven by the fact that during the past ten years, sensor prices have decreased by $50 \%$, bandwidth-related prices have reduced by more than $97 \%$ and processing prices have dropped by more than $98 \%$ [2].

With 25 billion devices expected to be part of IoT networks by 2020, much effort is put in designing solutions to network so many devices while maintaining good quality for the diverse supported services. Adaptive solutions considering different aspects for content delivery adjustment were proposed for video distribution over classic and heterogeneous network environments [3][4]. Quality of Service (QoS) levels and energy consumption are usually the metrics measured to verify the quality and efficiency of these services, which is especially important as IoT is often supported by a heterogeneous network environment [5][6].

The heterogeneity in IoT networking brings challenges

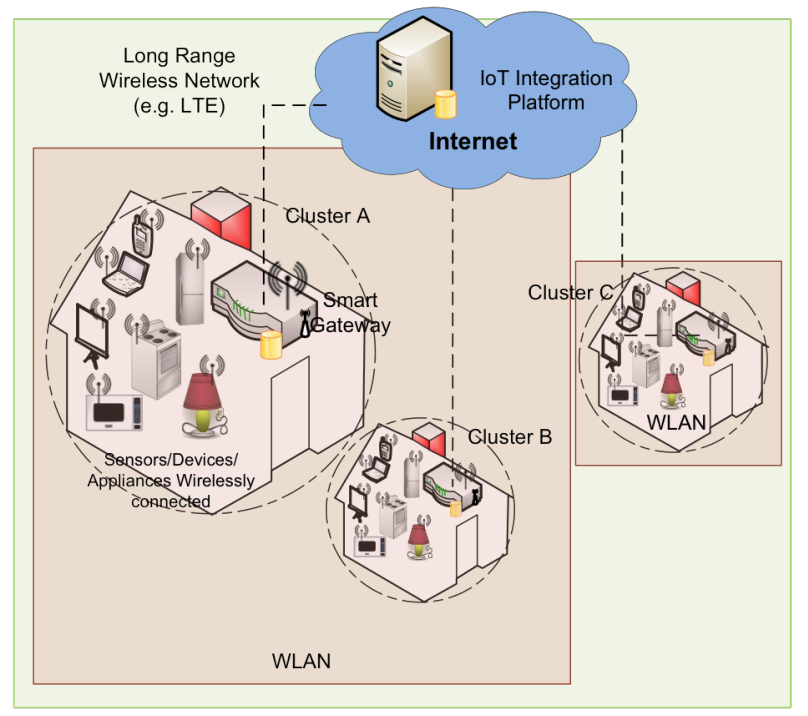

Fig. 1. NETSMITS's High Level IoT-based Architecture

regarding efficient data transmission, and the performance analysis of the different variables related to data transmission is thus of high importance.

This paper performs statistical analysis on QoS data when the innovative NETworking Scheme for sMart IoT gateways (NETSMITS) is employed to support increased quality for diverse IoT services, including video delivery [7]. The NETSMITS architecture is composed of clustered IoT objects, smart gateways, and the IoT Integration Platform (ITINP), as illustrated in figure 1. NETSMITS introduces an algorithm utilising both QoS metrics-related and service relevance scores in order to efficiently cluster interconnected IoT objects. The QoS metrics considered are throughput, delay and packet loss.

The statistical analysis on performance aims to detect what is the impact of network QoS metrics when IoT devices with different network requirements coexist.

The rest of this paper is organised as follows: section II introduces NETSMITS and discusses related works and section III introduces the statistical analysis, with section IV presenting the conclusions of this paper. 


\section{RELATED WORKS}

This section introduces NETSMITS and discusses other relevant IoT and QoS-based works relevant to NETSMITS performance assessment. These related research works are classified in two categories: IoT Background and IoT and QoS Statistical Analysis. These works demonstrate the importance of accurate QoS and QoE measurements for devices and applications, mainly in the realm of IoT, and how they can be used to improve network performance in execution time, which is the goal of NETSMITS.

\section{A. NETSMITS}

NETSMITS is an IoT solution [8] which increases the quality and performance of inter-communication between objects. The novelty of NETSMITS is associated with an algorithm which clusters the IoT objects efficiently around smart gateways by leveraging QoS and service relevance metrics.

The data generated by NETSMITS is analysed in this paper. NETSMITS block level components are illustrated in figure 2. The IoT objects' QoS Measurement Unit collects QoS metrics per each service provided by the object. The metrics are throughput, delay and packet loss ratio. The Smart Gateway's Admission Control Unit verifies if the gateway can support communication with a new object. The Smart Gateways' QoS Measurement Unit retrieves the QoS metrics (per service) for the objects that are not capable of running the algorithm themselves and also collects the smart gateways' related QoS metrics. The Decision Making Unit is in charge of regular computation of $Q o S$ and relevance scores, ranking of objects and deciding if they should be associated with another smart gateway or not. The ITINP Networking Scheme deploys an algorithm that receives a list of poor performing objects and attaches them to better suitable gateways. Significant improvements in performance were achieved by NETSMITS, with up to $63 \%$ more devices being supported and reduction of up to $85 \%$ in delay.

\section{B. IoT Background}

A novel feasibility study described in [9] evaluates the IEEE 802.11 ah protocol in terms of metrics such as the link budget, achievable data rate and packet size. IEEE 802.11ah is a new communications protocol focused on IoT, features a transmission range up to $1 \mathrm{~km}$ and data rates larger than 100 kbps.

The Energy-aware device-oriented adaptive multimedia scheme (eDOAS) [10], provides energy-aware adaptive streaming to devices in a heterogeneous WiFi and LTE network scenario. The adaptation is performed based on the values of various metrics including packet loss, throughput, Peak to Signal Noise Ratio (PSNR) and energy consumption. Similarly, cellular IoT has been described in [11].

The Multimedia IoT (MIoT), introduced in [12] is

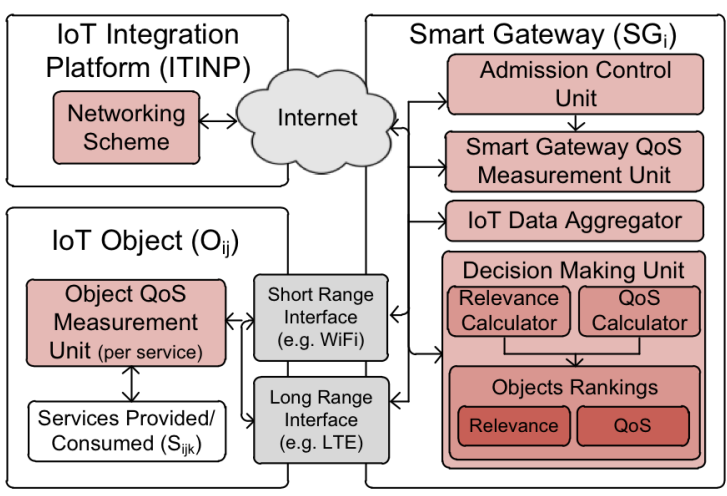

Fig. 2. NETSMITS architecture

presented through a vehicular application measured by the user Quality of Experience (QoE) metric.

In [13], a sensor cloud testbed with Adaptive QoS (AQoS) is presented, consisting of modules that offer diverse services. Different QoS models are analysed in Wireless Sensor Network (WSN) applications, based on historical data collected from a physical network. Reconfiguration of the cloud happens according to the result of the historic data performance analysis. QoS requirements are also analysed in [14], focusing on IoT networks enhanced by cognitive radio technology.

\section{IoT and QoS Statistical Analysis}

A number of papers have statistically analysed QoS in traditional and IoT networks.

In [15], the stochastic geometry theory is implemented to statistically analyse the random behaviour of IoT objects in femtocells and their impact on user QoS. Models generated from this analysis are used to formulate a heuristic algorithm for use in a two-level Stackelberg game. This game allows users to attempt to maximise their utilities while maintaining a balance. Such research follows a future trend towards optimising HetNet resource utilisation.

An analysis on QoS statistics over heterogeneous networks [16] generated important properties in terms of throughput, delay and jitter for both concise (minimum, maximum, average, median, standard deviation, and IQR values) and detailed (PDF, ACF, entropy, tail analysis, and bivariate PDF) statistics. Analysis concluded that parameters gathered at the edge of the network behaved differently from the ones gathered on the backbone, highlighting the usefulness of QoS statistics in identification of network elements and in anomaly detection frameworks.

The authors in [17] collected 8363 groups of data at endto-end wired multimedia transmission, selecting 4500 groups as training data and 5217 groups of data at end-to-end wireless multimedia transmission, selecting 3000 groups as training data. In applying an orthonormal algorithm, flow statistics such as packet delay, jitter, packet loss and frame rate became the input vector $\mathrm{x}$, while the output shows the 
one of four categories that the streaming is undertaking: normal transmission, a sender side CPU violation, a receiver side CPU violation and a congestion violation.

An analysis of variance (ANOVA) was performed in [18] and focused on QoS for IoT. The authors analysed three parameters in respect to collective QoS metrics for IoT Wireless Sensor Networks (WSN): collective latency, collective jitter and collective packet loss. Results show that by increasing the number of nodes in networks occupying smaller areas, packet loss is reduced, however for larger areas the packet loss increases with increased number of nodes.

\section{STATISTICAL ANALYSIS}

This section presents the IoT data collected, indicates how the data was measured, performs an analysis of the relationship among the QoS metrics and includes a comparison with another QoS statistical study. The analysis aims to identify the impact of network QoS metrics when IoT devices with different network requirements coexist.

\section{A. NETSMITS Data}

The data analysed was collected following modelling and simulation using the Network Simulator 3 (NS-3) [19]. Simulation parameters are presented in Table I. Simulationbased testing analysed the effect of the traffic from high bit rate devices, streaming high quality video which use most of the WiFi gateway network bandwidth, affecting overall system performance. As mentioned, NETSMITS includes IoT objects, network smart gateways and a cloud IoT Integration Platform (figure 1). NETSMITS also includes algorithms that calculate QoS scores and relevance of devices and based on these scores reallocates some of these devices to other gateways in terms of network attachment, improving the overall system delivery performance [8]. After applying NETSMITS, two devices that have most negatively impacted the system performance were attached to another gateway network.

In order to assess delivery QoS, at millisecond level, performance data is extracted from each device. The metrics measured are packet loss, delay and throughput. Packet loss is the percentage of packets (network communication units) that were sent by a source (e.g. server) and not received by the destination (e.g. IoT device). Delay is the time, expressed in milliseconds, required by the packets to reach their destinations. Throughput is the successful data rate a device was able to achieve in a certain amount of time, measured in this paper in Mbits/s (Mbps).

The data measured in each device is stored in files that contain the time of measurement expressed in milliseconds, bit rate (Mbps), throughput (Mbps), packet loss (percentage), delay (milliseconds), and device type.

The simulation ran for 30 seconds (300 milliseconds), however data was only exchanged from second 10 until second 20 , excluding any potential transitory periods.
TABLE I

SIMULATION SETUP

\begin{aligned} \hline Parameter & Value \\ Simulator & NS-3.24.1 \\ Duration of the Simulation & $10 \mathrm{~s}+10 \mathrm{~s}$ before and after sim. \\ WiFi Data Rate & $40 \mathrm{Mbps} \\$ WiFi Standard & $802.11 \mathrm{ac}(40 \mathrm{MHz}, \mathrm{MCS} 9) \\$ Distance between nodes and antennas & $3 \mathrm{~m} \\$ LTE eNB Antenna Model Type & Isotropic Antenna Model \\ Remote Station Manager & ConstantRateWifiManager \\ 4K Video Bit Rate & $25 \mathrm{Mbps} \\$ HDTV Bit Rate & $10 \mathrm{Mbps} \\$ SDTV Bit Rate & $4 \mathrm{Mbps} \\$ Other devices Bit Rate & $1 \mathrm{Mbps}\end{aligned}$

\section{B. SPSS and Device Groups}

The analysis was done in IBM SPSS Statistics, a software that helps to address the entire analytical process, from planning and data collection to analysis, reporting and deployment [20].

The simulation testbed devices which provides data for the analysis includes 8 devices, of four different types : 4K, $\mathrm{HD}$ and SD TVs, which receive data at $25 \mathrm{Mbps}, 10 \mathrm{Mbps}$ and $4 \mathrm{Mbps}$, respectively and another device type such as smart appliance or phone, video monitoring equipment, laptop, etc. receiving data at $1 \mathrm{Mbps}$.

\section{Descriptive Statistics and ANOVA Tests}

The tests in SPSS were based on the one-way ANOVA (Analysis of Variance). ANOVA was chosen after a good set of results was presented in [18], indicating it is a good statistical method for analysing QoS. One-way ANOVA was used because the metrics are directly compared against the

TABLE II

ANOVA TESTS

\begin{tabular}{|c|c|c|c|c|c|c|}
\hline & & $\begin{array}{c}\text { Sum of } \\
\text { Squares }\end{array}$ & df & $\begin{array}{c}\text { Mean } \\
\text { Square }\end{array}$ & $\mathbf{F}$ & Sig. \\
\hline \multirow{3}{*}{$\begin{array}{c}\text { Pct. } \\
\text { Achieved } \\
\text { of Max. } \\
\text { Thru. [\%] }\end{array}$} & $\begin{array}{c}\text { Between } \\
\text { Groups }\end{array}$ & 0.480 & 3 & 0.160 & 16.270 & 0.000 \\
\hline & $\begin{array}{l}\text { Within } \\
\text { Groups }\end{array}$ & 3.977 & 404 & 0.010 & & \\
\hline & Total & 4.457 & 407 & & & \\
\hline \multirow{3}{*}{ Delay [ms] } & $\begin{array}{c}\text { Between } \\
\text { Groups }\end{array}$ & 6.020 & 3 & 2.007 & 0.005 & 0.999 \\
\hline & $\begin{array}{l}\text { Within } \\
\text { Groups }\end{array}$ & 155194.647 & 404 & 384.145 & & \\
\hline & Total & 155200.667 & 407 & & & \\
\hline \multirow{3}{*}{$\begin{array}{c}\text { Packet } \\
\text { Loss[\%] }\end{array}$} & $\begin{array}{c}\text { Between } \\
\text { Groups }\end{array}$ & 0.075 & 3 & 0.025 & 28.760 & 0.000 \\
\hline & $\begin{array}{l}\text { Within } \\
\text { Groups }\end{array}$ & 0.352 & 404 & 0.001 & & \\
\hline & Total & 0.427 & 407 & & & \\
\hline
\end{tabular}


TABLE III

ANOVA MULTIPLE COMPARISONS

\begin{tabular}{|c|c|c|c|c|c|c|c|}
\hline \multirow{2}{*}{$\begin{array}{l}\text { Depend. } \\
\text { Var. }\end{array}$} & \multirow{2}{*}{$\begin{array}{l}\text { (I) } \\
\text { Dev. }\end{array}$} & \multirow{2}{*}{$\begin{array}{l}(J) \\
\text { Dev. }\end{array}$} & \multirow{2}{*}{$\begin{array}{c}\text { Mean } \\
\text { Diff. } \\
\text { (I-J) }\end{array}$} & \multirow{2}{*}{$\begin{array}{l}\text { Std. } \\
\text { Error }\end{array}$} & \multirow{2}{*}{ Sig. } & \multicolumn{2}{|c|}{$\begin{array}{l}\text { 95\% Confidence } \\
\text { Interval }\end{array}$} \\
\hline & & & & & & $\begin{array}{l}\text { Lower } \\
\text { Bound }\end{array}$ & $\begin{array}{l}\text { Upper } \\
\text { Bound }\end{array}$ \\
\hline \multirow{12}{*}{$\begin{array}{c}\text { Pct. } \\
\text { Achiev. } \\
\text { of Max. } \\
\text { Thru. } \\
\text { [\%] }\end{array}$} & \multirow{3}{*}{1} & 2 & -0.026 & 0.013 & 0.213 & -0.062 & 0.008 \\
\hline & & 3 & 0.024 & 0.013 & 0.310 & -0.011 & 0.059 \\
\hline & & 4 & 0.066 & 0.013 & 0.000 & 0.030 & 0.102 \\
\hline & \multirow{3}{*}{2} & 1 & 0.026 & 0.013 & 0.213 & -0.008 & 0.062 \\
\hline & & 3 & 0.050 & 0.013 & 0.002 & 0.015 & 0.086 \\
\hline & & 4 & 0.093 & 0.013 & 0.000 & 0.057 & 0.129 \\
\hline & \multirow{3}{*}{3} & 1 & -0.024 & 0.013 & 0.310 & -0.059 & 0.011 \\
\hline & & 2 & -0.050 & 0.013 & 0.002 & -0.086 & -0.015 \\
\hline & & 4 & 0.042 & 0.013 & 0.013 & 0.006 & 0.078 \\
\hline & \multirow{3}{*}{4} & 1 & -0.066 & 0.013 & 0.000 & -0.102 & -0.030 \\
\hline & & 2 & -0.093 & 0.013 & 0.000 & -0.129 & -0.057 \\
\hline & & 3 & -0.042 & 0.013 & 0.013 & -0.078 & -0.006 \\
\hline \multirow{12}{*}{$\begin{array}{l}\text { Delay } \\
\text { [ms] }\end{array}$} & \multirow{3}{*}{1} & 2 & 0.049 & 2.744 & 1.000 & $\begin{array}{l}-7.03 \\
\end{array}$ & 7.13 \\
\hline & & 3 & 0.069 & 2.744 & 1.000 & -7.01 & 7.15 \\
\hline & & 4 & -0.235 & 2.744 & 1.000 & -7.32 & 6.84 \\
\hline & \multirow{3}{*}{2} & 1 & -0.049 & 2.744 & 1.000 & -7.13 & 7.03 \\
\hline & & 3 & 0.020 & 2.744 & 1.000 & -7.06 & 7.10 \\
\hline & & 4 & -0.284 & 2.744 & 1.000 & -7.36 & 6.80 \\
\hline & \multirow{3}{*}{3} & 1 & -0.069 & 2.744 & 1.000 & -7.15 & 7.01 \\
\hline & & 2 & -0.020 & 2.744 & 1.000 & -7.10 & 7.06 \\
\hline & & 4 & -0.304 & 2.744 & 1.000 & -7.38 & 6.78 \\
\hline & \multirow{3}{*}{4} & 1 & 0.235 & 2.744 & 1.000 & -6.84 & 7.32 \\
\hline & & 2 & 0.284 & 2.744 & 1.000 & -6.80 & 7.36 \\
\hline & & 3 & 0.304 & 2.744 & 1.000 & -6.78 & 7.38 \\
\hline \multirow{12}{*}{$\begin{array}{c}\text { Packet } \\
\text { Loss[\%] }\end{array}$} & \multirow{3}{*}{1} & 2 & -0.010 & 0.004 & 0.047 & -0.021 & -0.000 \\
\hline & & 3 & -0.00 & 0.004 & 0.187 & -0.018 & 0.002 \\
\hline & & 4 & -0.036 & 0.004 & 0.000 & -0.046 & -0.025 \\
\hline & \multirow{3}{*}{2} & 1 & 0.010 & 0.004 & 0.047 & 0.000 & 0.021 \\
\hline & & 3 & 0.002 & 0.004 & 0.934 & -0.008 & 0.013 \\
\hline & & 4 & -0.025 & 0.004 & 0.000 & -0.036 & -0.014 \\
\hline & \multirow{3}{*}{3} & 1 & 0.008 & 0.004 & 0.187 & -0.002 & 0.018 \\
\hline & & 2 & -0.002 & 0.004 & 0.934 & -0.013 & 0.008 \\
\hline & & 4 & -0.028 & 0.004 & 0.000 & -0.038 & -0.017 \\
\hline & \multirow{3}{*}{4} & 1 & 0.036 & 0.004 & 0.000 & 0.025 & 0.046 \\
\hline & & 2 & 0.025 & 0.004 & 0.000 & 0.014 & 0.036 \\
\hline & & 3 & 0.028 & 0.004 & 0.000 & 0.017 & 0.038 \\
\hline
\end{tabular}

same metric on different devices. Device 1 is a $4 \mathrm{~K}$ TV with $25 \mathrm{Mbps}$ video bit rate, device 2 represents a HDTV with $10 \mathrm{Mbps}$ bit rate traffic, device 3 is an SDTV with $4 \mathrm{Mbps}$ video bitrate and device 4 represents general IoT devices with $1 \mathrm{Mbps}$ multimedia bit rate. There were 5 of those general devices in the testbed, and an average of their performance rates was taken. Figures 3 and 4 show the means of packet loss and throughput per device type with error bars indicating a confidence interval level of $95 \%$.

As the bit rate is different depending on the device, it would not be fair comparing the throughput of the devices, so for the analysis, we normalised the metric by retrieving the percentage of the maximum achievable throughput that the devices were able to achieve.

Table II shows the ANOVA results, which used the Tukey post hoc test with 0.05 significance. In terms of delay, having a 0.99 significance shows that there is no statistical difference

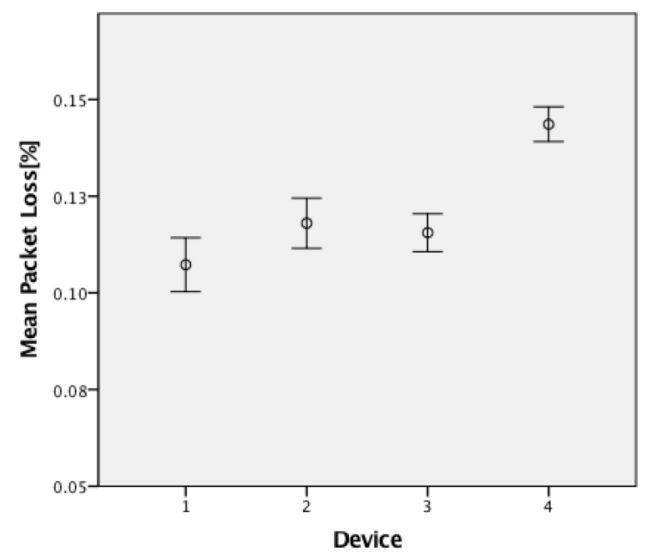

Fig. 3. Mean Packet Loss. Error bars: Confidence Interval level of $95 \%$

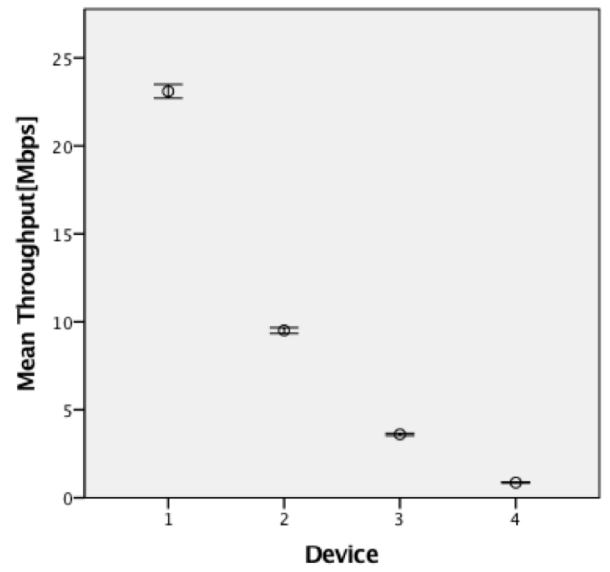

Fig. 4. Mean Throughput. Error bars: Confidence Interval level of $95 \%$

among the results associated with the different devices; however, for throughput and packet loss, which are below 0.05 , there is such a statistical difference.

The multiple comparisons results, presented in Table III, show that some objects had similar behaviour in the network, which might have affected the others.

Devices 2 and 3 had similar results in terms of packet loss, which show that, although all 4 devices had a similar average loss between $10 \%$ and $14 \%$, the distribution of loss during the simulation differed. Devices 2 and 3 had no significant difference between packet loss results, and neither devices 1 and 3. Devices 1 and 2 results show a small statistical difference. Device 4, which has low bit rate, is the one that suffered the most from packet loss and therefore there is a statistical difference between its results and those of the other three devices.

Regarding throughput, the devices that were most affected were devices 3 and 4 , again, the ones with lower bit rates. While between devices 1 and 2 , and devices 1 and 3 there are no statistical significant result differences, devices 2, 3 and 4 have statistical differences among them. This statistical analysis shows that the high bit rate devices receive higher bandwidth share and therefore benefit in terms of quality. 
Comparing this study with the analysis done in [18], similarities can be found. The authors used ANOVA to evaluate the quality of their novel model based on the Response Surface Methodology (RSM). The model uses a set of experiments to obtain optimal deployment parameters in respect to QoS metrics. Similar to the analysis done on NETSMITS data, a large number of nodes affected the overall performance. On NETSMITS, the large amounts of data consumed affected the performance of devices. In the RSM-based model, the probability of packet loss in a $5 \mathrm{~km}$ range varies from $0 \%$ for 100 low-power nodes to $60 \%$ for 900 low-power nodes.

\section{CONCLUSION}

This paper presents a statistical performance study of a recently proposed IoT networking solution, NETSMITS. The values of various metrics, such as bit rate and delay, throughput and packet loss are analysed in relation to the QoS measured on different device types.

Our findings show that delay is very similar in devices with different bit rate requirements, while throughput and packet loss affect mostly the low bit rate devices, giving an advantage to the high bit rate devices which is reflected in terms of quality. This analysis is extremely important for the development of efficient IoT solutions, such as algorithms for routers and gateways, that are expected to interconnect a large number of devices. These solutions will need optimal configurations and adaptability in terms of QoS metrics, in order to provide the best quality for the highest number of devices possible. A statistical method, such as ANOVA, could be applied in real time to IoT algorithms in order to guarantee that the best clustering tasks based on QoS metrics are performed.

Future works will involve implementing a statistical based approach into IoT solutions with a larger number of nodes to improve QoS further through pattern analysis and prediction during execution time. Such an approach will lead to more sophisticated and robust solutions in the growing area of IoT.

\section{ACKNOWLEDGMENT}

This work was supported by the Irish Research Council grant number EPSPG/2015/178 and in part by the European Union's Horizon 2020 Research and Innovation programme under Grant Agreement no. 688503 for the NEWTON project (http://newtonproject.eu).

\section{REFERENCES}

[1] J. Manyika, M. Chui, P. Bisson, J. Woetzel, R. Dobbs, J. Bughin, and D. Aharon, "Unlocking the potential of the Internet of Things", McKinsey, 2015. [Online]. Available: http://www.mckinsey .com/business-functions/digital-mckinsey/our-insights/the-internetof-things-the-value-of-digitizing-the-physical-world [Accessed: 02Dec-2016].

[2] Goldman Sachs, "The Internet of Things: Making sense of the next mega-trend", Goldman Sachs, 2014. [Online]. Available: http://www.goldmansachs.com/our-thinking/outlook/internet-ofthings/iot-report.pdf. [Accessed: 02-Dec- 2016].

[3] G. M. Muntean, P. Perry and L. Murphy, "Subjective Assessment of the Quality-Oriented Adaptive Scheme," in IEEE Transactions on Broadcasting, vol. 51, no. 3, pp. 276-286, Sept. 2005.

[4] G. M. Muntean, P. Perry and L. Murphy, "A Comparison-Based Study of Quality-Oriented Video on Demand," in IEEE Transactions on Broadcasting, vol. 53, no. 1, pp. 92-102, March 2007.

[5] A. Molnar and C. H. Muntean, "Cost-Oriented Adaptive Multimedia Delivery," in IEEE Transactions on Broadcasting, vol. 59, no. 3, pp. 484-499, Sept. 2013.

[6] R. Trestian, O. Ormond and G. M. Muntean, "Energy-Quality-Cost Tradeoff in a Multimedia-Based Heterogeneous Wireless Network Environment," in IEEE Transactions on Broadcasting, vol. 59, no. 2, pp. 340-357, June 2013.

[7] P. Biggs, J. Garrity, C. LaSalle, A. Polomska and R. Pepper, "Harnessing the Internet of Things for Global Development", International Telecommunication Union, 2016. [Online]. Available: https://www.itu.int/en/action/broadband/Documents/Harnessing-IoTGlobal-Development.pdf. [Accessed: 14- Mar- 2016].

[8] A. Simiscuka and G.-M Muntean, "A Networking Scheme for an Internet of Things Integration Platform," IEEE Int. Conf. Commun. Work. ICCW, 2017.

[9] A. Hazmi, J. Rinne, and M. Valkama, "Feasibility study of IEEE 802.11ah radio technology for IoT and M2M use cases," 2012 IEEE Globecom Workshops 2012, pp. 1687-1692, 2012.

[10] L. Zou, R. Trestian, and G. M. Muntean, "EDOAS: Energy-aware device-oriented adaptive multimedia scheme for Wi-Fi offload," IEEE Wirel. Commun. Netw. Conf. WCNC, pp. 2916-2921, 2014.

[11] A. Rachedi, M. Rehmani, S. Cherkaoui, and J. Rodrigues, "IEEE Access Special Section Editorial: The Plethora of Research in Internet of Things (IoT)," IEEE Access, vol. 4, pp. 9575-9579, 2016.

[12] A. Floris and L. Atzori, "Quality of Experience in the Multimedia Internet of Things: Definition and practical use-cases," 2015 IEEE Int Conf. Commun. Work. ICCW 2015, pp. 1747-1752, 2015.

[13] S. Ezdiani, I. S. Acharyya, S. Sivakumar, and A. Al-Anbuky, "An IoT Environment for WSN Adaptive QoS," 2015 IEEE Int. Conf. Data Sci. Data Intensive Syst., pp. 586-593, 2015.

[14] A. Khan, M. Rehmani, and A. Rachedi, "When Cognitive Radio Meets The Internet of Things?," Int. Wirel. Commun. Mob. Comput. Conf., pp. 469-474, 2016.

[15] R. Zhang, M. Wang, X. Shen and L. Xie, "Probabilistic Analysis on QoS Provisioning for Internet of Things in LTE-A Heterogeneous Networks with Partial Spectrum Usage," IEEE Internet of Things Journal, 3.3 (2016): 354-365.

[16] A. Botta, A. Pescapé, and G. Ventre, "Quality of service statistics over heterogeneous networks: Analysis and applications," Eur. Journal Oper. Res., vol. 191, no. 3, pp. 1075-1088, 2008.

[17] H. P. and L. N. L. Zhou, L. Chen, "Identifying QoS violations through statistical end-to-end analysis," Int. J. Commun. Syst., no. 24, pp. 1388-1406, 2011.

[18] G. Horvat, D. Žagar, and D. Vinko, "Influence of Node Deployment Parameters on QoS in Large-Scale WSN," IEEE MECO - 3rd Mediterr. Conf. Embed. Comput., no. 165, pp. 202-205, 2014.

[19] "The NS-3 network simulator." [Online]. Available: http://www.nsnam.org

[20] "IBM SPSS Statistics". [Online]. Available: http://www03.ibm.com/software/products/en/spss-statistics 